OBETS. Revista de Ciencias Sociales

Vol. 10, n. ${ }^{\circ} 1,2015$, pp. 185-209

ISSN: 1989-1385

DOI: 10.14198/OBETS2015.10.1.07

\title{
META PERIODISMO Y TRANSPARENCIA INFORMATIVA EN EL PERIODISMO DEL SIGLO XXI ${ }^{1}$ META-JOURNALISM AND MEDIA TRANSPARENCY IN JOURNALISM OF THE 21ST CENTURY
}

\author{
Eva Campos-Domínguez \\ Universidad de Valladolid, España \\ eva.campos@hmca.uva.es \\ Marta Redondo-García \\ Universidad de Valladolid, España \\ marta.redondo@hmca.uva.es
}

\begin{abstract}
Resumen
Esta investigación se enmarca dentro del periodismo postindustrial y pretende explorar la información que, sobre sí mismos, incluyen los medios de comunicación del modelo pluralista polarizado (Hallin y Mancini, 2004). Se analiza el grado de transparencia de las webs de seis medios: Italia (RAI-1 y Republica.it), Portugal (RTP-1 y Público.pt) y España (TVE-1 y Elpais.es). El estudio comparado atiende a una categorización desarrollada en base a Bardoel y d'Haenens, 2004; MediaACT, 2010, y Groenhart y Bardoel, 2012. Las conclusiones revelan que la transparencia comienza a difundirse en los sites aún de forma incipiente, de manera que la opacidad se mantiene como característica dominante frente a la rendición de cuentas.

Palabras clave: Periodismo abierto, Transparencia informativa, Cibermedios, Fiscalización mediática, Meta periodismo, Rendición de cuentas, Prosumidor.
\end{abstract}

\footnotetext{
${ }^{1}$ Esta investigación supone una ampliación y revisión de investigaciones previas presentadas por las autoras en el IV International Conference on Online Journalism, celebrado en la Universidade de Porto los días 4 y 5 de diciembre de 2014, así como en el XIV Congresso Internacional Ibercom 2015, celebrado en la Universidade de Sao Paulo los días 29 de marzo y 2 de abril de 2015. En esta versión ampliada se incorporan las discusiones realizadas a las comunicaciones presentadas durante sendos encuentros científicos.
} 


\begin{abstract}
This research is framed under the study of post-industrial journalism and explores the level of transparency in the Polarized Pluralist Model (Hallin \& Mancini, 2004). The paper analyzes the disclosure and accountability in six media web sites: Italy (RAI-1 and Republica.it), Portugal (RTP-1 and Publico.pt) and Spain (TVE-1 and Elpais.es). The methodological approach attends to a categorization that includes the media accountability systems built on the following contributions: Bardoel \& d'Haenens, 2004; MediaACT, 2010, and Groenhart \& Bardoel, 2012. The results show that transparency is beginning to spread in the sites but yet opacity seems to be the norm above the accountability.
\end{abstract}

Keywords: Open Journalism, Media Transparency, Cybermedia, Fiscalization, Meta-journalism, Accountability, Prosumer.

\title{
Extended abstract
}

The purpose of this research is to study transparency levels in the main news websites in the countries in Southern Europe. The paper analyzes the level of information given by the principal public television channels in Italy (RAI-1), Portugal (RTP-1) and Spain (TVE-1) and in the best-read newspapers, all of them private, of the three countries: Republica.it, Público.pt y Elpais.es., respectively.

The paper, within a methodological compared approach, attends to a categorization (of own elaboration) that includes the media accountability systems built on the following contributions: Bardoel \& d'Haenens, 2004; MediaACT, 2010, and Groenhart \& Bardoel, 2012. The research, situated in the context of post-industrial journalism, tries to raise the debate on media accountability in the Polarized Pluralist Model (Hallin \& Mancini, 2004) marked by a persistent disconnection among media power and the citizenship.

Mass media have not been unaware of the economic, social and political consequences of the crisis that, from the beginning of the 21 st century and with the advance of the postmodern era, confront the countries in Southern Europe. Since the 80 s, several researches try to investigate media changes that affect media economics, citizens disaffection and the revision process of the principles of journalism (Deuze, 2005), processes that have resulted in a crisis of media credibility (Barredo, 2013).

The paper analyzes if, in the new digital context, mass media advance towards an approach to the readers, recovering credibility in journalistic organizations, through meta-journalism, transparency and accountability.

Conclusions show little advance in media transparency in the main news sites (public and private) in the Polarized Pluralist Model (Hallin \& Mancini, 2004). The research shows that this progression is unequal, vertical and it is impossible to identify a clear media strategy in order to connect with citizenship to recover credibility trough transparency systems. The advance is unequal among public and private media, and among media in the different countries, and it cannot be concluded that in the countries where a culture of transparency has 
been introduced -through transparency laws- there is correlatively more transparency in media. It is especially relevant in television channels that are publicly funded and so transparency is an obligation for them as the regulation establishes but even though they don't satisfy fully the criteria of transparency contemplated in the theoretical models.

Though some media include transparency sections in their own web site, the information provided reveals a lack of a global strategy in order to advance in accountability in Southern Europe media (neither public nor private ones). For all this, it turns out clear that the analyzed media don't show a great commitment with the advance of transparency and open journalism: they don't disclosure enough information about the newsmaking process, they don't provide information in a way it could be re-usable, and don't make the most of the social capital that prosumers represent within the reporting process.

The analyzed websites do not include information about their way of working: scarcely have they included references to their codes of ethics, information about the media group owners and succinct references to the staff. The information they reveal about how they produce the information and quality controls are also an unknown.

Unlike experiences in other media that include links to videoconferences on the newsroom meetings to fix the daily agenda (The New York Times) or a newsroom blog that explains the criteria applied in selecting news (20minutos.es), the analysed media just show the finished work (news, report, coverage, etc.) but they do not explain the internal process which would provide the public with substantive content

Not even in the cases in which there are included substantive information (about credits, sales, benefits, results, etc.) the information is facilitated in a reusable format and data is mainly published in PDF format.

In no case do they use free license as a mean to share information with other media organizations but some of them promote the sales (is the case of Portuguese television which has a virtual store devoted to the sale on line of many products). In the same way, these media develop a vertical participation without interactive routes opened to the public, failing to take advantage of the possibilities of participation and public accountability systems in the site. So this is claimed that transparency is a mechanism for accountability but still it does not appear as an informative and ethical value in order to relieve the increasing disconnection between media organizations and the citizenship, and a system to regain credibility and public confidence.

Ultimately, the research concludes that, in spite of the civil demand of a major transparency in media organizations (press is a public service), the principal mass media of Italy, Spain and Portugal has a low level of open data journalism and recreate the old roles of the classic media. So they do not take advantage of the potential of transparency resources in order to gain credibility among the citizens and doing so they slow down the transformation that has to take place for a new way of journalism in the digital era. 


\section{INTRODUCCIÓN}

Los medios de comunicación no han sido ajenos a las consecuencias económicas, sociales y políticas de la crisis que, desde inicios del siglo XXI y con el avance de la posmodernidad, afrontan los países del sur de Europa. Desde los años 80 del siglo pasado, diversos académicos tratan de indagar en las razones de estos cambios mediáticos que afectan tanto a la estructura empresarial como a la desafección ciudadana y revisión de los principios del propio periodismo (Deuze, 2005), que han mermado la credibilidad de los medios de comunicación (Barredo, 2013) y plantean la necesidad del rescate del periodismo (Beckett, 2010).

La crisis mediática en los países de la Europa mediterránea adquiere unas características singulares, algunas de ellas ya señaladas por Hallin y Mancini (2004) al definir el modelo pluralista polarizado como aquél en el que destaca la integración de los medios de comunicación en la política de partidos, una tradición democrática tardía, y un nivel de profesionalización del periodismo también débil. En este orden, los autores señalan que los periódicos del sur de Europa estaban dirigidos a una pequeña élite, con contenidos politizados y con una tirada baja que se complementaba con una fuerte influencia de los medios audiovisuales en la opinión pública. En definitiva, este modelo característico de países como España, Italia y Portugal, partía de una concepción liberal de los medios de comunicación en el plano teórico, mientras que en la práctica su realidad se conformaba con un alto paralelismo político, entendido como mecanismo de influencia política y comercial.

Se produce una doble situación: por un lado, la búsqueda constante de la rentabilidad económica por parte de los medios de comunicación -en muchos casos avanzando, con este objetivo, hacia la frivolización de la información y entendiendo así los medios como espectáculo (Debord, 1968)- y por otro, una pérdida continuada de lectores debido, como señala Ramonet (2005), a la manipulación periodística, las relaciones entre los poderes financieros y mediáticos, y a la baja calidad de la información.

En este contexto, surge, a partir de la proliferación de Internet en los años 90 del siglo pasado, un nuevo agente que viene a transformar el paradigma comunicativo de los medios tradicionales y supone la transformación de los antiguos roles de emisor y receptor: la web 2.0 plantea un nuevo espacio en el que los usuarios consumen información, producen contenidos y se convierten en prosumidores (Toffler, 1980) a través de wikis, blogs y redes sociales como Facebook, Twitter, Flickr o YouTube (Chia, 2012). De esta forma, los nuevos emisores se transforman en un elemento hegemónico en este entorno comunicativo, replanteando las funciones tradicionales del emisor mediático (Berrocal, Campos y Redondo, 2014). 
El rol clásico del receptor, como un agente eminentemente pasivo en el proceso comunicativo, da lugar a un nuevo paradigma de creación de contenidos periodísticos donde los usuarios desempeñan un papel protagonista que García de Torres et al. (2009) tipificaron en tres categorías: el periodismo participante, donde los medios asumen la información creada por los ciudadanos; el periodismo ciudadano como una forma de comunicación amateur y no profesional, situada en la periferia de los medios de comunicación; y, finalmente, el contenido generado por el usuario como actividad comunicacional en la esfera digital y no necesariamente periodística (Barredo, 2013).

El papel cada vez más activo de los usuarios en el espacio comunicativo ha cuestionado la visión que, bajo el Cuarto Poder, ostentaba la prensa como defensora de la libertad de expresión y representante de la voz ciudadana ante los poderes políticos y económicos. Se sitúa así en el debate académico y profesional la pertinencia de reformular un discurso meta periodístico que abra el periodismo al escrutinio público y complemente la acción deontológica plasmada en protocolos profesionales de actuación del propio medio y regulada por organizaciones independientes, con la práctica colaborativa de la ciudadanía, al modo de un gran observador y fiscalizador constituido por los lectores, que abogue por incrementar los espacios dedicados a explicar el proceder del propio medio periodístico en la búsqueda, elaboración, redacción y difusión de la información (Oliveria, 2007) y, de esta forma, permita rescatar al periodismo (Beckett, 2010). Comienza, así, a hablarse de la necesidad de avanzar en el desarrollo de un periodismo transparente, pensando en la capacidad de los medios, con tres objetivos: para descubrir su forma de trabajar, para formular contenidos reutilizables y, en definitiva, para aprovechar el capital social del prosumidor para colaborar en el proceso informativo.

En este primer paso hacia la transparencia mediática, los medios actuarían ofreciendo información de sí mismos: mostrando quiénes son, cómo construyen la información, en definitiva, contando su forma de trabajar (Regina, 2008). Anderson, Bell y Shirky (2013) refieren el periodismo postindustrial como aquel en el que los medios de comunicación dan cuenta de los procesos que usan para crear periodismo de calidad, mediante mecanismos de transparencia y sistematización, mostrando su trabajo para que ese proceso sea tanto observado y entendido por los lectores, como reutilizado por otros medios y organizaciones periodísticas. En este contexto es en el que se aprecia un desarrolo más tímido en los países con menor tradición democrática y periodística, con una mayor inseguridad profesional en el sector considerando que si se revela la back-information se estaría ayudando a la competencia y perjudicando al medio. Sin embargo los mismos autores afirman: «no vemos ninguna razón por la que 
las organizaciones no puedan seguir ganando dinero y obteniendo primicias en esta nueva era, incluso enseñando la forma de trabajar» (Anderson, et al., 2013:34).

En un segundo nivel de desarrollo del periodismo transparente, las organizaciones informativas estarían proveyendo información reutilizable para la ciudadanía y otras instituciones periodísticas, creando así un contexto informacional construido en base a unos principios objetivos de cooperación y sustentabilidad. En esta fase cabría referir también al capital social para colaborar en el proceso informativo, incluyendo la posibilidad de asociaciones con otras organizaciones para proporcionar información o conocimiento y avanzando tanto hacia el Cuarto Poder en red (Sampedro, 2014) como hacia la integración, dentro de los procesos periodísticos, de la evaluación de impacto por parte de esta ciudadanía como integrante de la cultura organizacional, mediante la fiscalización ciudadana de cada medio y la monitorización de datos.

En este contexto de periodismo postindustrial, cabría plantear, en efecto, el debate en torno a la información que sobre sí mismos incluyen los medios de comunicación del modelo pluralista polarizado (Hallin y Mancini, 2004) marcados por una acuciante desconexión entre los poderes mediáticos y la ciudadanía, y con ello plantear si la nueva esfera comunicativa en el espacio digital permite que los medios puedan avanzar hacia una mayor cercanía con sus lectores, recuperando la credibilidad (Barredo, 2013), y tomando como eje de actuación el meta periodismo, mediante la transparencia informativa y la rendición de cuentas.

\section{TRANSPARENCIA Y ORGANIZACIONES MEDIÁTICAS}

El interés público por la transparencia como valor democrático surge tras la Primera Guerra Mundial en el proceso negociador que siguió al conflicto (Braman, 2006). No obstante, la proliferación de Internet y el actual contexto de desafección ciudadana y crisis institucional, ha situado nuevamente en el debate académico y profesional la pertinencia de la rendición de cuentas, permitiendo la distribución y acceso de datos con un coste mínimo.

Tal y como señala Guichot (2011), ya en el año 2011, en el Libro blanco La Gobernanza europea se diagnosticó una acusada pérdida de confianza de los ciudadanos en las instituciones, y se propuso, entre sus líneas de actuación, el fomento de un mayor grado de apertura y participación, facilitando información a través de Internet sobre el desarrollo de las políticas en todas las etapas del proceso de elaboración. La transparencia se considera, por tanto, uno de los instrumentos más eficaces para promover y restaurar la confianza de la sociedad en las instituciones públicas que permite garantizar la legitimidad de las administra- 
ciones y favorecer una participación más informada de los ciudadanos en las decisiones que les afectan (Bhatnagar, 2003; Shim y Eom, 2008; Anderson, 2009; Bertot, Jaeger y Grimes, 2012).

Las distintas aportaciones académicas coinciden en definir la transparencia como la apertura de las organizaciones al escrutinio público sobre sus decisiones y funcionamiento interno. Por ejemplo, Ugalde explica el concepto como la «práctica de colocar la información en la vitrina pública para que aquellos interesados puedan revisarla, analizarla y, en su caso, usarla como mecanismo para sancionar en caso de que haya anomalías en su interior» (2002:17) y, por su parte, Florini la identifica con «el grado de información disponible que permite a los ciudadanos que están fuera tener una opinión informada y/o evaluar las decisiones tomadas por quienes están dentro» (2007:5). De esta forma, el concepto de transparencia se relaciona directamente con el de accountability o rendición de cuentas que supone la obligación de explicar y justificar la conducta, lo que implica no solo el aporte de información suficiente sino también el sometimiento al examen y debate públicos, y, ocasionalmente, la imposición de sanciones (Bovens, 2005).

En Europa, la velocidad de incorporación a una «cultura de la transparencia» ha sido irregular. Considerando como indicador de transparencia institucional, las leyes que permiten el acceso a buena parte de su información y a dar respuesta a las cuestiones planteadas por la ciudadanía, se observa que los países pioneros en la aprobación de estas normas fueron Suecia en 1766, Finlandia en 1951, y Dinamarca y Noruega en 1970, mientras que se sitúan a gran distancia el resto de países que mayoritariamente han incorporado este tipo de leyes a su ordenamiento a finales de los años 90 del siglo XX y en los primeros años del siglo XXI (Guichot, 2011).

En este sentido, Italia, cuenta con una Ley de Transparencia aprobada desde 1990 con la finalidad de mejorar la colaboración entre los ciudadanos y la administración pública, y desde entonces ha sufrido ya varias modificaciones (la última, en 2009). Cuenta, además, con la Comisión Independiente por la Valorización, Transparencia e Integridad de las Administraciones Públicas (CIVIT) que actúa como organismo garante de su cumplimento. Por su parte, Portugal aprobó en 1993 la Ley de acceso a los documentos de la administración (LADA) que fue sustituida en 2007 por la Ley de acceso a los documentos administrativos y su reutilización. Además, dispone de una Comisión de Acceso a Documentos Administrativos (CADA), una entidad independiente que vela por el cumplimiento de las leyes relativas al derecho de acceso.

El caso español es el más tardío de los tres, y ha sido uno de los últimos estados europeos en aprobar una ley de estas características. El proceso se ha 
desarrollado en un contexto de «importante discusión y presión transformadora en la opinión pública» (Dader, Campos-Domínguez y Quintana, 2011:13), abanderado también por el movimiento $15 \mathrm{M}$ y «Democracia Real Ya!» como una de sus reivindicaciones. La Ley de transparencia, acceso a la información pública y buen gobierno fue aprobada en 2013, aunque hasta diciembre de 2014 no entró en vigor para los organismos estatales y hasta diciembre de 2015 para los organismos autonómicos y locales.

Al margen de las iniciativas legales, existe un creciente movimiento ciudadano que promueve un aumento de la transparencia institucional y que se canaliza a través de organizaciones cívicas como Transparencia Internacional o Access Info Europe, cuyo objetivo es concienciar a los ciudadanos sobre las consecuencias de la corrupción y presionar para que exista un mayor control de gobiernos e instituciones. Esta corriente ha venido también estimulada por las reacciones a la crisis económica mundial, entendiendo que ésta había tenido su origen «en errores fundamentales en relación con la transparencia, la rendición de cuentas y la responsabilidad» (Parlamento Europeo, 2012).

Adicionalmente, diversos estudios interpretan que la transparencia de lo público es insuficiente si las corporaciones privadas no se abren también al examen de los ciudadanos. Fung y Weil (2010) mantienen que de la transparencia de las instituciones públicas se debe pasar a la sociedad abierta dado que las amenazas para los individuos y para la sociedad en su conjunto provienen tanto, o quizá más que de los gobiernos, del poderoso sector privado que controla sectores tan influyentes como la banca, los productores de alimentos o la industria farmacéutica.

Los medios de comunicación no son ajenos a esta necesidad de una mayor transparencia y se están sumando a la cultura de la rendición de cuentas como fórmula para ganar credibilidad en un entorno en el que se está renegociando el contrato social del periodismo (Eide, 2014), dado que necesita volver a legitimarse ante la aparición de importantes competidores que producen información y comunicación aunque ésta no sea profesional. Según el código de buenas prácticas del MediaAct (2012), la transparencia mediática ayuda a incrementar la calidad del periodismo y facilita una relación de confianza con la audiencia, creando un mejor diálogo entre el público y los medios de comunicación.

En este contexto, la interactividad, como elemento identificador de los nuevos medios de comunicación (Shultz, 2006; Hujanen y Pietikäinen, 2004), pasa porque la audiencia pueda exigir información y los medios se sientan obligados a responder a las cuestiones que versan sobre la labor periodística. De hecho, como recoge Smolkin (2006), la transparencia mediática se ha situado en el foco del debate público en Estados Unidos al trascender distintos escándalos 
ocurridos en medios prestigiosos porque sus periodistas publicaron informaciones falsas poniendo en evidencia el fallo de los sistemas de control internos -los casos Jayson Blair o Judith Miller en The New York Times o Stephen Glass en The New Republic-. Aunque los errores cometidos no suponían ninguna novedad, sí era inédita la existencia de una generación de blogueros especializados en la crítica de los medios y de lectores concienciados que exigían respuestas y asunción de responsabilidades.

Otros autores han puesto de manifiesto la necesidad de articular mecanismos que garanticen la transparencia mediática como instrumento de fiscalización para la prensa y como una nueva forma de relacionarse con la audiencia (Kovach y Ronsenstiel, 2003; McQuail, 2003). Un proceso que, para McBride y Rosenstiel, está avanzando por la presión de los receptores: «se acabaron los días en los que los consumidores confiaban en las noticias simplemente porque eran producidas por una casta de profesionales. Ahora determinamos si una información es fiable buscando la evidencia de su credibilidad» (2014: 217).

En este sentido, se entiende la relevancia de la transparencia como un elemento coadyuvante en la mejora del periodismo de calidad y de su inexcusable obligación de verificación: «La única manera práctica de explicar al público lo que sabemos es mostrar nuestros métodos y fuentes en el grado máximo que sea posible» (Kovach y Ronsenstiel, 2003: 126). Karlsson, Clerwall y Nord (2014) otorgan una mayor importancia a la trasparencia mediática e interpretan que sus posibilidades actuales de desarrollo pueden conducir a un cambio de paradigma en el periodismo, de forma que se sustituiría el principio supremo de la objetividad -cuya responsabilidad recae únicamente en el informadorpor el de la transparencia-compartida entre productor y receptor, es decir: el prosumidor mediático-, otorgando capacidad a la audiencia para valorar por sí misma el grado de rigor presente en el trabajo informativo.

La mayoría de los autores entiende que el nivel de transparencia en los medios debe ser elevado con escasos elementos ocultos sobre el quehacer periodístico, de modo que ayude a entender al receptor la importancia de la función informativa y su nivel de fiabilidad: «parece evidente que cada vez más se requiere de mecanismos que le faciliten al público la compresión del continuum periodístico: cómo se seleccionan los hechos que pasarán a formar parte del menú informativo a partir del cual nos nutriremos o desnutriremos; las razones por las que hechos diversos se incorporan o se dejan de lado en la agenda temática de cada medio; el significado de los compromisos periodísticos pactados (la protección de fuentes confidenciales, por ejemplo); los procesos de producción de las informaciones; las razones para optar por campos y géneros periodísticos determinados a la hora de narrar un suceso; las implicancias de 
las fuentes de primera, segunda y tercera mano, el on y el off the record; la cómplice ausencia textual de algunos actores; el significado de la edición, y la presencia de las ilustraciones y la elocuencia del diseño» (Freundt-Thurne, 2005: 13).

Los mecanismos de transparencia permiten al medio, de esta forma, explicar públicamente el trabajo periodístico. De cara al público, contribuyen a mejorar su alfabetización mediática de forma que comprenda el funcionamiento de la prensa, sus limitaciones y trascendencia, generando receptores más conscientes y críticos (Regina Maia, 2008). Aquellos consumidores interesados en la actividad interna de los medios pueden obtener una información de mayor calidad estableciendo relaciones de significado entre la noticia y sus condiciones de producción (Freundt-Thurne, 2005). Pero también resulta útil como mecanismo de control de las organizaciones que gestionan buena parte de los medios de comunicación y que acumulan poder y capacidad de influencia (Guerrero, 2008).

\section{SISTEMATIZACIÓN DE LA TRANSPARENCIA MEDIÁTICA}

Las distintas organizaciones internacionales encargadas de valorar la transparencia han utilizado una metodología de análisis basada en la elaboración de índices de requisitos a cumplir y su posterior verificación para otorgar una puntuación a cada organismo, entidad o empresa. Transparencia Internacional, por ejemplo, establece una serie de ítems y ofrece una calificación teniendo en cuenta el grado de consecución de los requisitos establecidos. Un sistema similar se aplica en diversos estudios realizados sobre medios de comunicación. El proyecto europeo Media Accountability and Transparency in Europe (MediaACT) realiza un estudio comparativo sobre el desarrollo de las fórmulas de transparencia implantadas por los medios de comunicación en distintos países de Europa con el objetivo de articular recomendaciones a la Unión para que incentive el desarrollo de estos sistemas de control y estimule la concienciación de los usuarios. España, sin embargo, sólo interviene como miembro asociado, a pesar de haber participado en el desarrollo del estado de la cuestión (Alsius, Mauri y Rodríguez, 2011).

Los mecanismos para analizar la transparencia mediática han sido categorizados por diversos autores, incluso se han acuñado los acrónimos MAIs: Media Accountability Instruments o MAS: Media Accountability Systems para identificar «cualquier medio no público (no estatal) de responsabilizar a los medios respecto de su audiencia» (Claude-Jean Betrand, 2000: 18).

La categorización más básica es la que establece MediaACT. Atendiendo al momento de aparición de los mecanismos de transparencia, los clasifica en 
mecanismos tradicionales que comprenderían los existentes en la etapa analógica como códigos éticos, consejos de prensa, ombudsman, cartas al director o secciones de comunicación de los medios, y mecanismos innovadores como las webs sobre medios, los blogs de periodistas y defensores de la audiencia, los comentarios de los lectores o la información en redes sociales.

Bardoel and d'Haenens (2004), refiriéndose a la rendición de cuentas de los medios, diferencian cuatro dimensiones de la transparencia: la relativa a las políticas de comunicación, el mercado, el ejercicio profesional y el público. Groenhart y Bardoel (2012), por su parte, desarrollan un modelo en el que distinguen entre la transparencia en la producción -production transparencyrelativa a cómo se obtienen y elaboran las noticias, que incluiría la información sobre fuentes consultadas e intereses de éstas; las técnicas de newsgathering, o la posibilidad de publicar material complementario sobre el que se construye la historia o meta información; la transparencia de los participantes, o actores-actor transparency-, referida tanto al periodista como a la empresa y que comprendería información sobre la propiedad del medio y sus fuentes de financiación, las normas de funcionamiento interno del medio o la información sobre la plantilla de trabajadores y, finalmente, la transparencia de la interlocución -dialogue transparency-, que pone su foco en la audiencia y se aplicaría a las relaciones de interactividad que el medio mantiene con su público.

El International Center for Media and the Public Agenda (ICMPA) en su estudio sobre la transparencia en las webs de 25 medios de Estados Unidos, Reino Unido y Oriente Medio, realizado en el año 2006, particulariza aún más las categorías de análisis y establece las siguientes cinco variables: por un lado, correcciones, que responde a la apertura y responsabilidad del medio a la hora de reconocer públicamente y rectificar los errores que se hayan cometido en la elaboración de las informaciones; en segundo lugar, la propiedad, que afecta a quienes poseen el medio y sus intereses empresariales o estratégicos; en tercer lugar, la política de personal, referida a las normas internas que guían el trabajo de los periodistas, editores o productores; en cuarto lugar, política informativa, sobre la transparencia en las decisiones editoriales y los procesos de producción de la información y, finalmente, la interactividad, que valora la apertura a la hora de recibir y difundir los comentarios y las críticas de los receptores.

\section{METODOLOGÍA}

El objetivo de esta investigación es medir el grado de transparencia mediática en tres países incluidos dentro del modelo pluralista polarizado de Hallin y Mancini (2004), tomando como objetivo de estudio los sitios web de una muestra representativa de medios de financiación pública y privada. De esta forma, se 
analiza la transparencia mediática en Italia, Portugal y España, tomando como casos de estudio las siguientes organizaciones periodísticas: las cadenas de televisión públicas Rai-1, RTP y TVE-1, así como las siguientes empresas de financiación privada: La Republica.it, Publico.pt y elpais.es. Se corresponden, respectivamente, con las principales cadenas públicas de televisión en cada país, y con los periódicos más leídos y consultados en Internet en cada uno de ellos (ComScore, 2013).

En última instancia, se pretende verificar si estos medios de comunicación están desarrollando la transparencia mediática en base a los tres objetivos referidos previamente: para descubrir su forma de trabajar, para formular contenidos reutilizables y, en definitiva, para aprovechar el capital social del prosumidor para colaborar en el proceso informativo.

Se entiende que la diferencia en la tradición de la cultura de transparencia en cada uno de los países analizados, mediante la aprobación de leyes de transparencia estatales, unidos a las características del modelo pluralista polarizado propio de estos tres países, permitirá diagnosticar si la transparencia es, o no, un mecanismo de desarrollo en estos medios para la rendición de cuentas y, en último término, si se presenta como valor informativo y deontológico para tratar de paliar la creciente desconexión entre las organizaciones mediáticas y la ciudadanía, y recuperar su credibilidad y confianza.

La hipótesis principal que se intenta verificar es que la tradición de la cultura de transparencia, a través de las leyes de transparencia públicas, influye en un mayor grado de transparencia mediática y, por tanto, las organizaciones periodísticas italianas estudiadas presentarán un mayor grado de desarrollo de la transparencia que las españolas o portuguesas. Sin embargo, como subhipótesis, se considera que, aún en este caso, los avances hacia el periodismo transparente serán tímidos en estas organizaciones analizadas a la hora de descubrir su forma de trabajar, para formular contenidos reutilizables y para aprovechar el capital social del prosumidor como colaborador en el proceso informativo.

Se aplica un análisis de contenido de las webs de los seis medios de comunicación referidos, mediante una revisión realizada durante los meses de agosto, septiembre y octubre de 2014 y actualizada en enero y febrero de 2015. El análisis fue aplicado por dos codificadoras que individualmente testaron la presencia o ausencia de los indicadores en las webs, en colaboración con investigadores y alumnos de intercambio para el caso de los sites portugueses e italianos en el marco de trabajo del Grupo de Investigación «GIR-Nuteco» de la Universidad de Valladolid con el objetivo de interpretar correctamente las webs mediáticas de los respectivos países y minimizar los errores culturales y lingüísticos. Una vez rastreadas las webs, las investigadoras realizaron una interpretación 
conjunta de cada una de las variables estudiadas en cada cibermedio, para minimizar los errores interpretativos intercodificadores.

Las variables observadas han atendido a una categorización de elaboración propia que incluye los mecanismos que posibilitan el control externo, desarrollada tomando como fundamento las aportaciones de Bardoel y d'Haenens, 2004; MediaACT, 2010, y Groenhart y Bardoel, 2012, señaladas en el epígrafe anterior. El desglose y propuesta de las categorías e ítems fueron presentados en dos congresos científicos internacionales de periodismo, donde se testó su validez y correspondencia con los objetivos. En la presente investigación se incluyen las modificaciones y sugerencias planteadas como debate en dichos encuentros. Las variables se miden con dígitos, y se aplica, también, una interpretación cualitativa de los ítems observados.

El instrumento de análisis es, por tanto, una lista de comprobación o checklist desglosada en 38 ítems agrupados en tres categorías, y aplicado sobre un baremo de 0 a 2 puntos en cada ítem, donde 0 equivale a ítem totalmente ausente y 2 totalmente presente:

Por un lado, se observa la categoría de transparencia económica -en la que se incluyen 9 ítems-, que incluye parámetros relativos a la propiedad del medio y a la gestión empresarial del mismo tales como información sobre el grupo de comunicación al que pertenece, la composición accionarial del mismo, la relación de anunciantes y la inversión que realiza cada uno o la cantidad que se ingresa por publicidad institucional. El objetivo de esta categoría de transparencia es conocer cómo se financian los medios y en qué niveles esas fórmulas pueden afectar a su función periodística.

Por otro lado, se atiende a la transparencia editorial -en la que figuran 14 ítems-. Remite tanto a la información sobre las normas internas del medio como sobre su organización y organigrama. En este punto se valoran aspectos como la aparición explícita de la línea editorial del medio, el acceso a su libro de estilo y a su código ético, la existencia de ombusman, la disponibilidad de información sobre corrección de errores o información sobre el organigrama y el currículum de los miembros de la plantilla. El objetivo de esta categoría es detectar cómo producen información los medios, desde el punto de vista de ejercicio profesional.

Adicionalmente, se contempla la transparencia de la interactividad-que abarca 15 ítems-. Analiza las fórmulas de participación de los lectores y el diálogo que establecen con el medio. En este punto, el estudio distingue entre dos fórmulas de participación: por un lado, la participación de arriba abajo (del medio al usuario) que se subdivide en: pasiva, interpelativa, consultiva y deliberativa (Campos-Domínguez, 2011), dependiendo del grado de intervención del 
usuario, desde la simple elección de contenidos (por ejemplo, suscripción a contenidos RSS) hasta la posibilidad de intervenir en un diálogo plural dentro del medio (por ejemplo, a través de los comentarios a las informaciones); $y$, por otro lado, la participación de abajo arriba (del usuario al medio) que se refiere a la capacidad del usuario de tener su propio espacio de contenidos opinativos o informativos independientes dentro de la web del medio.

Se trata, en definitiva, de un análisis exploratorio de carácter preliminar, que no incluye parámetros de transparencia en la producción de la información dado que exige distinta metodología de investigación consistente en el análisis de una selección de informaciones publicadas en los medios con el fin de estudiar aspectos tales como el uso de las fuentes, los métodos de obtención de información o el volcado de materiales en bruto.

\section{RESULTADOS}

La transparencia mediática en las organizaciones periodísticas analizadas es todavía materia pendiente y presenta un escaso desarrollo en su escala general: en ninguno de los casos observados se aprecia una presencia dominante de aquellos elementos que se consideran, según los referentes teóricos, definitorios de una transparencia mediática.

Por lo que se refiere a la categoría de transparencia económica (Tabla 1) se analiza la web respecto a la información facilitada sobre la propiedad del medio, los resultados económicos y la dependencia de entidades financieras, y valora la información que ofrece sobre los ingresos por publicidad y la procedencia de la misma, fuentes de financiación, estado de las cuentas, entendiendo que la claridad en la gestión económica facilita, aunque no garantice, la calidad del servicio público de la información (Jones, 2007; Kovach y Rosenstiel, 2003).

En este sentido, en los parámetros contemplados se aprecia que la mayoría de los medios incluyen información exhaustiva relativa a la propiedad de la organización, fundamentalmente para el caso de los medios de financiación pública (televisiones), pero sus niveles de cumplimento de transparencia son muy bajos para el resto de elementos, fundamentalmente en lo referido a la información sobre las fuentes de ingresos de cada medio.

La mayoría de las cadenas de televisión -medios de financiación públicadisponen de una sección de transparencia denominada «Transparencia» para el caso de $\mathrm{TVE}^{2}$ o bien, «Amministrazione trasparente», en la $\mathrm{RAI}^{3}$ en la que se

\footnotetext{
${ }^{2}$ Información disponible en http://www.rtve.es/corporacion/transparencia/ (consultado el 01/03/2015)

${ }^{3}$ Información disponible en: http://www.rai.it/dl/rai/text/ContentItem-e5f6f732-clc349b3-ba62-0c085c1609db.html (consultado el 01/03/2015)
} 
incluye información económica y estadística; licitaciones, contratos y demás información administrativa y jurídica; así como también informes de auditorías y organigrama. En todos estos casos o bien la información es ofrecida en formato no-reutilizable -imágenes, archivos en formato .pdf o similares- o bien parte de las secciones se encuentran incompletas o en construcción; por tanto, la información ofrecida sobre esta materia es, hasta la fecha, escasa.

Las fuentes de ingresos, rendimientos por publicidad o patrimonio $\mathrm{u}$ otros recursos es un aspecto mayoritariamente no revelado. En algunos casos, como por ejemplo para el caso de los medios públicos italianos o españoles, se ofrece información sobre los presupuestos y partidas generales, los datos no se encuentran en ningún caso desglosados. Llama incluso la atención para el caso de la información sobre las tarifas publicitarias o venta de producciones propias, que es oculta para la mayoría de los medios analizados - a excepción de El País-y en el caso de los medios públicos tampoco se publican las tarifas para ventas de productos, con la única salvedad de la tienda de la televisión portuguesa que ofrece la venta online de variados productos -desde libros, hasta alojamientos de hotel ${ }^{4}-$.

Tabla 1. Transparencia económica

\begin{tabular}{|c|c|c|c|c|c|c|c|}
\hline & $\begin{array}{r}\mathbf{D} \\
\text { El } \\
\text { Pais }\end{array}$ & $\begin{array}{l}\text { ID } \\
\text { Público }\end{array}$ & $\begin{array}{c}\text { Rfit } \\
\text { República }\end{array}$ & $\begin{array}{l}\text { tve.es } \\
\text { TVE-1 }\end{array}$ & $\begin{array}{l}\equiv \\
\text { RTP-1 }\end{array}$ & Rai 1 & Prom \\
\hline \multicolumn{8}{|l|}{ Información sobre la propiedad del medio } \\
\hline Grupo de comunicación al que pertenece & 1 & 1 & 2 & 2 & 2 & 2 & 1,7 \\
\hline Composición accionarial & 1 & 2 & 1 & 2 & 2 & 2 & 1,7 \\
\hline Cuentas de resultados anuales & 1 & 0 & 1 & 2 & 2 & 2 & 1,3 \\
\hline \multicolumn{8}{|c|}{ Fuentes de ingresos. Publicidad y ayudas públicas } \\
\hline Relación de anunciantes publicitarios & 0 & 0 & 0 & 1 & 1 & 1 & 0,5 \\
\hline Inversión realizada por anunciante & 0 & 0 & 0 & 0 & 0 & 0 & 0,0 \\
\hline Tarifas publicitarias del medio & 2 & 2 & 0 & 0 & 0 & 0 & 0,7 \\
\hline Publicidad institucional & 0 & 0 & 0 & 0 & 0 & 0 & 0,0 \\
\hline \multicolumn{8}{|c|}{ Dependencia crediticia: organismo y cantidad } \\
\hline Deudas crediticias: organismo y cantidad & 1 & 2 & 1 & 1 & 1 & 1 & 1,2 \\
\hline Auditorias / mecanismos externos & 1 & 2 & 1 & 1 & 1 & 1 & 1,2 \\
\hline Total (Máx. 18) & 7 & 9 & 6 & 9 & 9 & 9 & 8,2 \\
\hline
\end{tabular}

Fuente: Elaboración propia. Datos relativos a septiembre y octubre de 2014 y a enero y febrero de 2015. Sombreado en gris valores de variable parcialmente presente $(\mathrm{v}=1)$ en negro, variable complementa presente $(\mathrm{v}=2)$ y en blanco, variable ausente $(\mathrm{v}=0)$

${ }^{4}$ Información disponible en: http://www.lojartp.pt/ (consultado el 01/03/2015) 
En el caso de información sobre dependencia crediticia, auditorías y mecanismos de control externo la transparencia es parcial porque, a excepción del diario portugués Publico.pt, en la mayoría de las organizaciones periodísticas observadas apenas se ofrece información general, y en ningún caso se plantea un detalle del procedimiento de las auditorias, ni se ofrecen datos en bruto.

En cuanto a la transparencia editorial (Tabla 2), esta categoría remite a la información sobre las normas internas que rigen en el medio y a su organización y organigrama. En este punto se valoran aspectos como el acceso al libro de estilo, al código ético o a la línea editorial, la disponibilidad del currículum vítae de los miembros que componen la plantilla y la exposición de sus responsabilidades. Incluye además el ideario editorial de un medio, lo que permite conocer el enfoque de un determinado evento ante su cobertura (Smolkin, 2006).

Así, la transparencia editorial cumple también con escasos parámetros del total de los analizados. La más completa es la información editorial en la que el medio detalla su ideario, explica su grupo de comunicación, etc.; seguido por la información explícita de la línea editorial del medio. En contra, aquellos parámetros relacionados directamente con el proceso de redacción y elaboración periodística -cómo el medio realiza información de calidad-son los que se encuentran más ocultos -libro de estilo, descripción profesional de la plantilla del medio, estatutos de redacción, etc.-, así como también los elementos vinculados directamente con la rendición de cuentas con los lectores -corrección de errores-. En este sentido, es significativo señalar que ninguno de los medios analizados publica un histórico de las correcciones de errores satisfechas de forma visible en la web, ni tampoco información actual sobre las correcciones, y la información relativa a cómo solicitar una rectificación es muy limitada para todos los medios.

Es importante destacar que la información relativa a los códigos deontológicos de los medios -principalmente internos- está presente total o parcialmente en todos los medios analizados, así como especialmente destacable es la importancia que los medios portugueses y españoles destinan, en su sitio, al defensor del lector reservando secciones específicas para esta figura con información detallada sobre sus funciones y actuaciones, así como herramientas para fortalecer el intercambio de pareceres entre el propio defensor de estos medios y los lectores. Llaman, no obstante, la atención los medios italianos al no publicar en sus sitios web ninguna referencia a esta figura ni a sus actuaciones; mientras que en el caso de la televisión italiana sí se halla, a diferencia del resto de los medios, un blog del director del medio, aunque éste está destinado fundamentalmente a la opinión y no a la transparencia de la actividad de la organización ${ }^{5}$.

\footnotetext{
${ }^{5}$ Información disponible en http://zucconi.blogautore.repubblica.it/ (Consultado el 3 de abril de 2015)
} 
Tabla 2. Transparencia editorial (organizacional y periodística)

\begin{tabular}{|c|c|c|c|c|c|c|c|}
\hline & $\mathbf{E}$ & IP & Rift & tre.es & 仼 & Rai 1 & \\
\hline & El Pais & Público & República & TVE-1 & RTP-1 & RAI-1 & Prom \\
\hline Información editorial & 1 & 1 & 2 & 2 & 2 & 2 & 1,7 \\
\hline Línea editorial del medio & 2 & 2 & 1 & 1 & 1 & 2 & 1,5 \\
\hline Principios Editoriales & 0 & 2 & 1 & 1 & 1 & 1 & 1,0 \\
\hline Libro o Manual de Estilo & 0 & 0 & 0 & 0 & 0 & 0 & 0,0 \\
\hline Estatuto de Redacción & 0 & 0 & 1 & 2 & 0 & 0 & 0,5 \\
\hline Otros códigos internos & 2 & 1 & 1 & 2 & 2 & 2 & 1,7 \\
\hline Códigos externos & 0 & 0 & 0 & 1 & 0 & 0 & 0,2 \\
\hline Ombusman & 2 & 2 & 0 & 2 & 2 & 0 & 1,3 \\
\hline Blog /SRS de Ombusman & 2 & 2 & 0 & 2 & 2 & 0 & 1,3 \\
\hline Corrección de errores & 0 & 0 & 0 & 1 & 1 & 0 & 0,3 \\
\hline Información solicitar corrección & 1 & 0 & 0 & 1 & 1 & 0 & 0,5 \\
\hline Histórico corrección errores & 0 & 0 & 0 & 0 & 0 & 0 & 0,0 \\
\hline Organigrama & 1 & 2 & 1 & 2 & 2 & 1 & 1,5 \\
\hline Datos profesionales de plantilla & 0 & 2 & 0 & 0 & 0 & 0 & 0,3 \\
\hline Total (Máx. 28) & 11 & 14 & 7 & 17 & 14 & 8 & 11,8 \\
\hline
\end{tabular}

Fuente: Elaboración propia. Datos relativos a septiembre y octubre de 2014 y a enero y febrero de 2015. Sombreado en gris valores de variable parcialmente presente ( $v=1)$ en negro, variable complementa presente $(\mathrm{v}=2)$ y en blanco, variable ausente $(\mathrm{v}=0)$

Pese a la unanimidad en cuanto a la importancia de la transparencia de la interactividad, buena parte de las investigaciones considera que el desarrollo interactivo aún resulta insuficiente de cara a conseguir que los medios fortalezcan la discusión pública y generen un sistema ciudadano de vigilancia que propicie una democracia realmente deliberativa: «aún queda la sensación de que hay muchas cosas que el nuevo periodismo debería de estar haciendo para monitorear, responsabilizar, facilitar y mantener la deliberación» (Fenton, 2010: 5). Como asegura Frankenber, a menudo los sitios web «proclaman su interés en ofrecer interacción a sus usuarios, pero a su vez procuran con cautela no debilitar el dogma del periodismo moderno de: nosotros escribimos, ustedes leen» (2015: 108).

El análisis que ocupa a este estudio confirma esta tendencia: a pesar de que el grado de transparencia es relativamente alto para los niveles de participación pasiva, en los que el usuario únicamente actúa en la selección de contenidos que desea recibir, se aprecia en la tabla 3 que a mayor protagonismo del ciudadano, menos espacios disponibles en los medios. Es resaltable, además, que en la mayoría de los casos los medios incluyen herramientas que permiten la participación -por ejemplo, enlaces a redes sociales- pero en escasas ocasiones se seña- 
lan las condiciones de participación y compromiso del medio con esta actividad del lector: únicamente los medios españoles dedican un espacio a explicitar las condiciones de esta contribución ${ }^{6}$.

En la mayoría de los medios observados los ciudadanos pueden seleccionar los contenidos que desean consultar bien a través de la suscripción de contenidos RSS, bien a través de la suscripción de contenidos personalizada a través de correo electrónico o bien a través del boletín electrónico. No se contabilizan en este categoría las posibilidades que el usuario puede tener de búsqueda de

Tabla 3. Transparencia de la interactividad

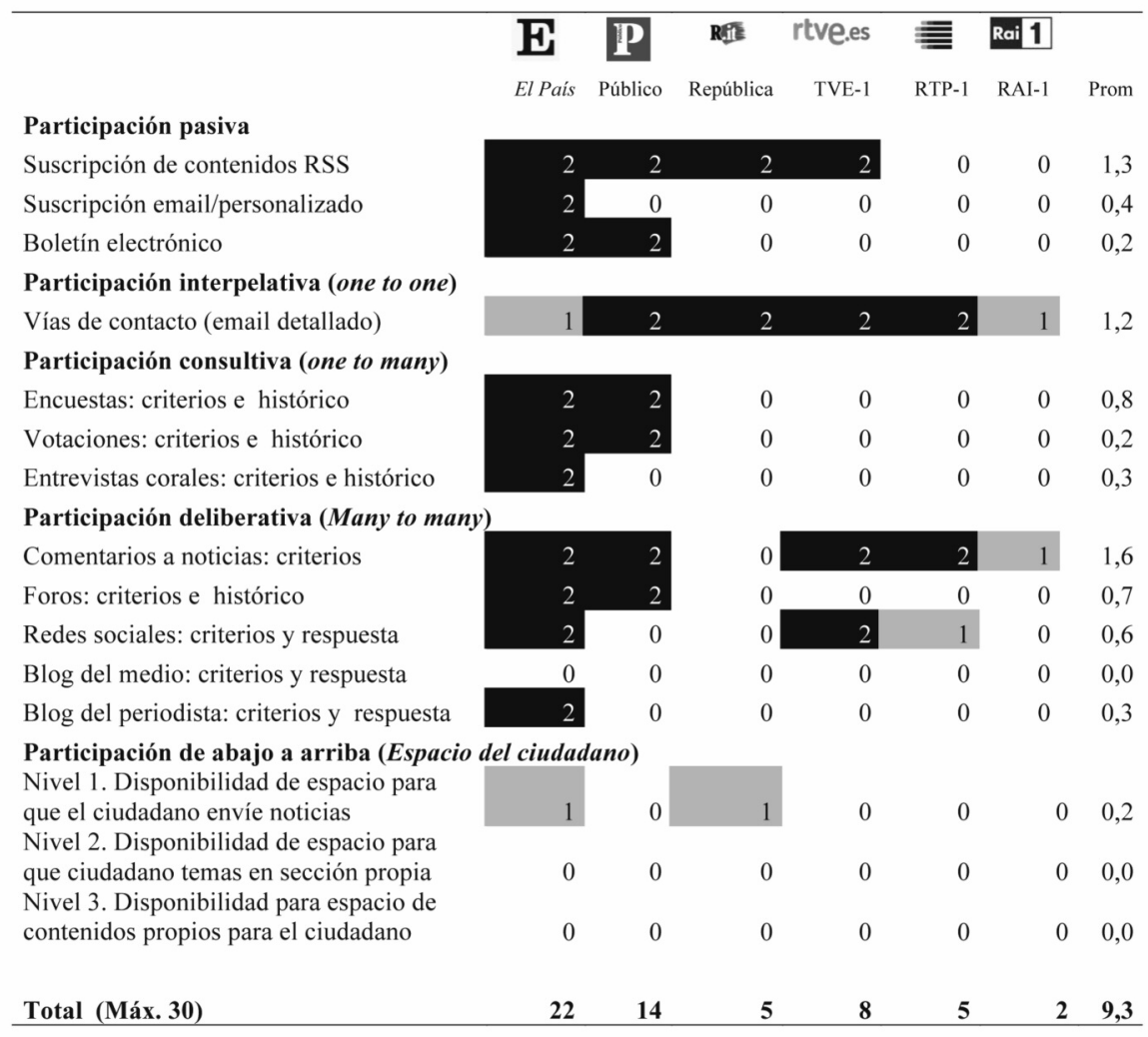

Fuente: Elaboración propia. Datos relativos a septiembre y octubre de 2014 y a enero y febrero de 2015. Sombreado en gris valores de variable parcialmente presente $(v=1)$ en negro, variable complementa presente $(\mathrm{v}=2)$ y en blanco, variable ausente $(\mathrm{v}=0)$.

${ }^{6}$ Véase por ejemplo: http://www.rtve.es/comunes/normas_participacion.shtml (Consultado el 5 de marzo de 2015) 
información en el propio site, si esta información no es enviada al lector -en esta situación estaría, por ejemplo, el espacio de TVE «televisión a la carta»-. Para el caso de los medios públicos (televisiones) se aprecia la tendencia mayoritaria de consultar y reproducir información en su propio espacio web, sin que ésta pueda ser enviada al usuario de forma personalizada.

La transparencia en las herramientas de participación bidireccional está presente parcial o totalmente en todos los medios, no obstante en ninguno se ofrece información sobre el tráfico de correos intercambiados entre los lectores y el medio o con los periodistas. Por otro lado, las herramientas de participación consultiva tradicionales -como encuestas, foros, etc.- han sido sustituidas, en la mayoría de los casos, por enlaces a redes sociales, consolidándose así la tendencia a una comunicación más propia del prosumidor mediático -que reproduce y viraliza la información del medio- que del activista -que fiscaliza la información del medio-. En ningún caso se ofrece tampoco información de los criterios de participación y compromiso del medio con la respuesta o contenidos elaborados por los ciudadanos en estos espacios de participación.

La opacidad en términos de información sobre el tráfico de comunicación entre el lector con el medio y/o periodista es absoluta en todos los casos: ningún medio informa del número de correos que recibe de lectores, cuántos usuarios reciben boletines electrónicos, tampoco se detalla información sobre número y contenido de mensajes intercambiados, ni el funcionamiento de la participación consultiva ni deliberativa.

Por lo que se refiere a la transparencia mediática clasificada en categorías -económica, editorial y de la interactividad- (Tabla 4) representa también un escenario general de escaso desarrollo: la económica, que está presente en un mayor número de elementos de los sitios web analizados, supone un promedio de 45,4 ítems satisfechos en el conjunto de las webs, frente a la de la interactividad, con los niveles más opacos de las tres categorías atendidas (30,3 puntos).

Tabla 4. Nivel general de transparencia mediática

\begin{tabular}{|c|c|c|c|c|c|c|c|}
\hline $\begin{array}{l}\text { Datos \% de } \\
\text { transparencia }\end{array}$ & $\mathbf{E}$ & IP & Rili & rtve.es & 言 & Rai 1 & \\
\hline & El Pais & Público & República & TVE-1 & RTP-1 & RAI-1 & Prom \\
\hline Económica & 38,9 & $\mathbf{5 0 , 0}$ & 33,3 & $\mathbf{5 0 , 0}$ & $\mathbf{5 0 , 0}$ & $\mathbf{5 0 , 0}$ & 45,4 \\
\hline Editorial & 39,3 & $\mathbf{5 0 , 0}$ & 25,0 & 60,7 & 50,0 & 28,6 & 39,3 \\
\hline \multirow[t]{2}{*}{ De interactividad } & 73,3 & 46,7 & 16,7 & 26,7 & 16,7 & 6,7 & 30,9 \\
\hline & $\mathbf{5 0 , 5}$ & 48,9 & 25,0 & 45,8 & 38,9 & 28,4 & 38,5 \\
\hline
\end{tabular}

Fuente: Elaboración propia. Datos relativos a septiembre y octubre de 2014 y a enero y febrero de 2015. Destacado en negrita valores más altos y bajos para cada categoría. 
Dentro de los valores generales de escasa transparencia (Tabla 5), se observan ligeras diferencias en la presencia de variables en función de los medios públicos estudiados -cadenas de televisión de financiación estatal-, frente a los medios de financiación privada -diarios digitales-. Así, en el promedio general los medios de comunicación privados obtienen un nivel de cumplimiento de transparencia mayor que los públicos ( 41,5 puntos porcentuales frente a 37,7 , respectivamente) esta diferencia se explica porque, aunque las cadenas de televisión alcanzan puntuaciones más altas en la económica y editorial, la diferencia en la transparencia de la interactividad es de casi cuatro puntos porcentuales más en los medios privados que en los públicos, lo que desequilibra los niveles de transparencia generales.

Tabla 5. Promedio de transparencia mediática por tipo de medio y país

\begin{tabular}{|c|c|c|c|c|c|}
\hline \multirow{2}{*}{$\%$ Transparencia } & \multicolumn{2}{|c|}{ Por tipo } & \multicolumn{3}{|c|}{ Por pais } \\
\hline & Privados & Públicos & España & Portugal & Italia \\
\hline Económica & 40,7 & 50,0 & 44,4 & 50,0 & 41,7 \\
\hline Editorial & 38,1 & 46,4 & $\mathbf{5 0 , 0}$ & $\mathbf{5 0 , 0}$ & 26,8 \\
\hline De interactividad & 45,6 & 16,7 & 50,0 & 31,7 & 11,7 \\
\hline Promedio total & 41,5 & 37,7 & 48,1 & 43,9 & 26,7 \\
\hline
\end{tabular}

Fuente: Elaboración propia. Datos relativos a septiembre y octubre de 2014 y a enero y febrero de 2015. Datos en \% promedio de ítems satisfechos por tipo de medio y país. En negrita y sombreados en gris los valores mayores para cada categoría de transparencia.

Se observa, asimismo, que ningún promedio supera, ni por país ni por tipo de transparencia, el 50\% de los puntos establecidos, por lo que se concluye que el nivel de opacidad en los medios analizados es todavía significativo. Las diferencias localizadas son llamativas para el caso italiano respecto de los medios portugueses y españoles: a pesar de que Italia cuenta con una mayor tradición en la ley de transparencia estatal, los medios de los otros dos países han atendido de forma más intensa a los elementos de transparencia.

\section{CONCLUSIONES}

Se aprecia un tímido avance hacia la transparencia mediática en los principales medios de financiación pública y privada de Italia, España y Portugal vinculados al modelo pluralista polarizado, teorizado por Hallin y Mancini (2004); no obstante, se concluye que esta progresión es desigual, verticalizada y sin que pueda localizarse una estrategia clara de conexión por parte de la organización periodística con la ciudadanía para recuperar la credibilidad mediática a través de estos mecanismos de rendición de cuentas. 
El avance es diferente entre los medios públicos y privados, según cada caso, y entre los propios países, sin que pueda concluirse que una mayor cultura de la transparencia -a través de la formalización de las leyes estatales de transparencia- influya correlativamente en una mayor transparencia de los medios. Especialmente llamativo es el caso de las cadenas de televisión -medios de financiación pública-, sujetos a la obligatoriedad de transparencia que se contempla en la normativa, y que tampoco satisfacen plenamente los criterios de transparencia contemplados en los referentes teóricos.

Aunque algunos medios incluyen una sección denominada "Transparencia" en su propio sitio web, la información allí publicada revela una falta de estrategia colectiva para avanzar en esta área: no existe una inquietud colectiva por parte de los medios de comunicación de la Europa del Sur -ni para los públicos ni para los privados- que permitan discutir estándares de cumplimento.

Por todo ello, resulta claro que los intereses explícitos que los medios analizados muestran hacia el avance de la transparencia y del periodismo abierto deslocalizan los objetivos planteados a la hora de descubrir información de su forma de trabajar, para la formulación de contenidos reutilizables y para aprovechar el capital social del prosumidor en el proceso informativo.

En efecto, los medios de comunicación observados no incluyen información sobre su forma de trabajar: apenas facilitan referencias a sus códigos éticos, información de su grupo editorial y referencias escuetas a su plantilla, pero los datos que pueden descubrir cómo el medio realiza esa información para valorar su calidad son todavía una gran incógnita profesional y mediática. A diferencia de experiencias con tradición y vocación de transparencia de otros medios que incluían, por ejemplo, conexiones en videoconferencias sobre las reuniones de la sala de redacción para señalar la agenda del día (The New York Times) o un blog de redacción en el que se señalan los criterios para la búsqueda de noticias y conformación del portal del medio (20minutos.es), los medios observados únicamente ofrecen los contenidos finales -noticias, reportajes, crónicas, etc.- sin explicar el proceso de elaboración interno de las mismas.

En la misma línea, ni siquiera en los casos en los que se incluyen datos informativos relacionados, por ejemplo, con la transparencia económica, éstos se presentan en formato reutilizable, siendo la tónica general los datos publicados en imágenes o .pdf. En ningún caso se contemplan licencias libres como opciones para compartir información con otras organizaciones periodísticas, y más bien se potencia la venta del producto -incluso para el caso del medio televisivo portugués, mediante una tienda virtual de venta de productos diversosfrente a la colaboración, consolidándose así la comunicación verticalizada. De la misma forma, esta opacidad se muestra también en la interactividad con el 
usuario, desaprovechando las posibilidades de participación mediante mecanismos de rendición de cuentas públicos en el site.

Se afirma, por tanto, que la transparencia es, explícitamente, un mecanismo de desarrollo en estos medios para la rendición de cuentas pero todavía no se presenta como valor informativo y deontológico para tratar de paliar la creciente desconexión entre las organizaciones mediáticas y la ciudadanía, y recuperar su credibilidad y confianza.

En definitiva, el análisis realizado permite concluir que, a pesar de la demanda ciudadana de una mayor transparencia en las organizaciones públicas y de servicio público -como tradicionalmente se ha entendido el papel de la prensa- los principales medios de comunicación de Italia, España y Portugal satisfacen bajos niveles de transparencia y periodismo abierto, repitiendo todavía roles empresariales y periodísticos de los medios clásicos, sin que ello pueda servir para avanzar hacia una mayor credibilidad ciudadana en las organizaciones mediáticas y ralentizando la transformación que la situación actual requiere para la conformación de la rendición de cuentas en la recuperación del Periodismo.

\section{REFERENCIAS}

Alsius, S.; Mauri, M. y Rodríguez, R. (2011). "Spain: A diverse and asymmetric landscape". En Eberwein, T.; Fengler, S.; Lauk, E.; Leppik-Bork, T. (Eds.), Mapping Media Accountability - in Europe and Beyond. Colonia, Halem.

Anderson, T. B. (2009). "E-government as an anti-corruption strategy". Information Economics and Policy, 21, 201-210.

Anderson, C.W.; Bell, E. y Shirky, C. (2013). Periodismo postindustrial: adaptación al presente. Tow Center For Digital Journalism / Columbia Journalism School. Disponible en: http://www.amic.media/media/files/file_352_441.pdf Consultado: 20/04/2015)

Bardoel, J. y d'Haenens, L. (2004). "Media responsibility and accountability: New conceptualizations and practices". Communications, 29, vol. I, 5-25.

Barredo, I. (2013). "La crisis de credibilidad de las organizaciones periodísticas: hacia la sociedad de los ideantes". Poliantea, IX (16), 101-126

Beckett, C. (2010). "Más cerca del ciudadano activo. El rescate del periodismo". Infoamérica: Iberoamerican Communication Review, 2, 45-57.

Berrocal, S.; Campos, E. y Redondo, M. (2014). "Prosumidores mediáticos en la comunicación política: El «politainment» en YouTube", Comunicar, 43, V. XXII, 65-72

Bertot, J.C., Jaeger, P. T. y Grimes, J.M. (2012). "Promoting transparency and accountability through ICTs, social media, and collaborative e\#government". Transforming Government: People, Process and Policy, 6 (1), 78-91. 
Bertrand, C-J. (2000). Media Ethics and Accountability Systems. Cresskill, NJ: Hampton Press.

Bhatnagar, S. (2003). E-government and access to information. Global Corruption Report 2003. Washington: Transparency International.

Bovens, M. (2005). "Public Accountability: A framework for the analysis and assessment of accountability arrangements in the public domain". En Ferlie, E., Lynne, L. y Pollitt, C. (Eds.). The Oxford Handbook of Public Management, Oxford: Oxford University Press.

Braman, S. (2006). Change of state: Information, policy, and power. Cambridge: Massachusetts Institute of Technology Press.

Campos-Domínguez, E. (2011). La ciberdemocracia en el Congreso de los Diputados (2004-2008). Madrid: Congreso de los Diputados.

Chia, A. (2012). "Welcome to Me-Mart". American Behavioral Scientist, 56 (4), 421-438

Dader, J. L., Campos-Domínguez, E. y Quintana, N. (2011). "Las webs de los partidos en la campaña de 2011: Una panorámica cualitativa de su actividad y un análisis cuantitativo de su transparencia”. En: Crespo Martínez, I. (Dir.) Partidos, medios y electores en proceso de cambio: las elecciones generales españolas de 2011. Valencia : Tirant Humanidades, 239-274.

Debord, G. (1967). La société du spectacle. París: Buchet/Chastel.

Deuze, M. (2005). "What is journalism? Professional identity and ideology of journalists reconsidered". Journalism, 6(4), 442-464.

Eide, M. (2014). "Accounting for Journalism”. Journalism Studies, 15(5), 679-688.

Fenton, N. (2010), "Drowning or waving? New media, journalism and democracy", en N. Fenton (Ed.), New media, old news. Journalism E Democracy in the Digital Age. Londres, SAGE, 3-16.

Florini, A. (2007). The Right to Know. Transparency for an Open World. Nueva York: Columbia University Press.

Freundt-Thurne, U. (2005), "Periodismo y visibilidad. El laberinto de la transparencia", Cuadernos de información, 18, 12-21.

Frankenberg, Lorena (2015). "Interactividad y democracia deliberativa en el periodismo digital en México". Comunicación y Sociedad (nueva época), 23, 97-123.

Fung, A. y Weil, D. (2010). "Open Government and Open Society". En: Lathrop, D. y Ruma, L. (Eds.) Open Government: Collaboration, Transparency, and Participation in Practice. Cambridge: O'Reilly, 105-113.

García de Torres, E.; et al. (2009). "UGC Status and levels of control in Argentine, Colombian, Mexican, Peruvian, Portuguese, Spanish, US and Venezuelan online newspapers. International Symposium on Online Journalism. Austin (Texas). Disponible en: https://online.journalism.utexas.edu/2009/papers/Garciaetal09.pdf Consultado: 21/04/2015)

Groenhart, H. y Bardoel, J. (2012). "Conceiving the transparency of journalism; towards a new media accountability currency". Studies in Communications Sciences, 12, vol. I, 6-11 
Guerrero, M. A. (2008). Medios de comunicación y la función de la transparencia. México: Instituto Federal de Acceso a la Información Pública.

Guichot, E. (2011). Transparencia y acceso a la información pública en España: análisis y propuestas legislativas. Madrid: Fundación Alternativas.

Hallin, D. C. y Manicini, P. (2004). Comparing Media Systems. Three Models of Media and Politics, Nueva York: Cambridge University.

Hujanen, J. y Pietikäinen, S. (2004). "Interactive Uses of Journalism: Crossing Between Technological Potential and Young People's News-Using Practices". New Media E Society, 6 (3), 383-401.

Jones, D. (2007). "Grupos mediáticos y culturales en España", ZER, 22, 183-214.

Karlsson, M.; Clerwall, C. y Nord, L. (2014), "You Ain’t Seen Nothing Yet: Transparency's (Lack of) Effect on Source and Message Credibility". Journalism Studies, $15,5,668-678$.

Kovach, B. y Rosenstiel, T. (2003). Los elementos del periodismo. Madrid: Aguilar.

McBride, K. y Rosenstiel, T. (Eds.) (2014). The New Ethics of Journalism: Principles for the 21st Century. Londres: SAGE.

McQuail, D. (2003). Media Accountability and Freedom of Publication. Oxford: Oxford University Press.

MediaAct (2012). Best Practices Book: Media Accountability and Transparency across Europe. Austria: Institute of Journalism and Communication of the University of Tartu.

Oliveria, M. (2007). Metajornalismo... ou quando o jornalismo é sujeito do próprio discurso. Tese de doutoramento. Universidade do Minho. Disponible en: http://hdl.hand le.net/1822/7035 (Consultado: 21/04/2015)

Ramonet, I. (2005). "Medios de comunicación en crisis". Le Monde diplomatique. Disponible en: http://www.lemondediplomatique.cl/medios-de-comunicacionen-crisis.html (Consultado: 21/04/2015)

Regina Maia, M. (2008). "The rule of transparency: as an element of democratization in journalism production process". Brazilian Journalism Research, vol. IV, 2, 119-138.

Shim, D. C. y Eom, T. H. (2008). "E-government and anti-corruption: Empirical analysis of international data". International Journal of Public Administration, 31, 298-316.

Shultz, T. (2006). "Interactive Options in Online Journalism: A content Analysis of 100 U.S. Newspapers". Journal of Computer-Mediated Communication, 5, 1. Disponible en: http://onlinelibrary.wiley.com/doi/10.1111/j.1083-6101.1999.tb003 31.x/full (Consultado: 19/04/2015)

Smolkin, R. (2006). "Too Transparent?", American Journalism Review, 28, vol. II, 16-23. Toffler, A. (1980). The Third Wave. Nueva York (USA): Bantam Books.

Ugalde, LC. (2002). Rendición de cuentas y democracia. El caso de México. México: Instituto Federal Electoral. 
EVA CAMPOS DOMÍNGUEZ. Profesora de Periodismo en la Universidad de Valladolid (Titular Acreditada). Su área de especialización es la Comunicación en Internet. Doctora en Comunicación y Premio Extraordinario de Doctorado de la Universidad Complutense de Madrid. Becaria de las Cortes Generales para la elaboración de Tesis Doctorales (2007 y 2008) y Premio Joven de Comunicación de la Fundación General de la Universidad Complutense (2007). Ha realizado estancias de investigación postdoctoral en diferentes centros de investigación internacionales, y participado en diferentes proyectos de investigación competitivos. Sus líneas de investigación se centran en: Comunicación Política, Comunicación digital, Ciberperiodismo y Comunicación legislativa.

MARTA REDONDO GARCÍA es Profesora Ayudante Doctora de Periodismo en la Universidad de Valladolid. Es Premio Extraordinario de Doctorado y Primer Premio Internet de Periodismo de la Junta de Castilla y León. Miembro del Grupo de Investigación Reconocido Nuevas Tendencias en Comunicación. Ha ejercido como redactora y presentadora en distintos canales de televisión: Antena 3, Telemadrid (Vía Digital) y Televisión Española, entre otros, donde ha trabajado en programas informativos. Su investigación se ha centrado en el análisis del sensacionalismo informativo, el infoentretenimiento y la Transparencia Mediática.

Recibido: 19/05/2015

Aceptado: 20/06/2015 